\title{
Erzhi Formula Extracts Reverse Renal Injury in Diabetic Nephropathy Rats by Protecting the Renal Podocytes
}

\author{
Jun Jiang $\left(\mathbb{D},{ }^{1}\right.$ Jiangning Yin, ${ }^{2,3}$ Xiang Liu, ${ }^{1}$ Huajun Wang, ${ }^{2}$ and Guoyuan $\mathrm{Lu}^{3}$ \\ ${ }^{1}$ School of Pharmacy, Jiangsu University, No. 301 Xuefu Road, Zhenjiang, Jiangsu 212013, China \\ ${ }^{2}$ Affiliated Hospital of Jiangsu University, No. 438 Jiefang Road, Zhenjiang, Jiangsu 212001, China \\ ${ }^{3}$ The First Affiliated Hospital of Soochow University, No. 188 Shizi Road, Suzhou, Jiangsu, 215006, China
}

Correspondence should be addressed to Jun Jiang; jiangjuntcm2007@hotmail.com

Received 2 April 2018; Revised 28 July 2018; Accepted 5 August 2018; Published 23 August 2018

Academic Editor: Yuewen Gong

Copyright (C) 2018 Jun Jiang et al. This is an open access article distributed under the Creative Commons Attribution License, which permits unrestricted use, distribution, and reproduction in any medium, provided the original work is properly cited.

\begin{abstract}
Podocytes injury was a crucial factor resulting in diabetic nephropathy (DN). Erzhi formula extract (EZF) was a clinical effective Chinese medicine on DN, but its mechanism was unclear. In this study, the main compounds of EZF and their pharmacokinetics in rat were detected by HPLC-MS/MS. And then, blood glucose, urine protein, renal index, renal microstructural (HE/PAS staining), inflammatory factors (IL- $\beta$, TNF- $\alpha$, IL-6), and protein/mRNA expression related to the function of podocyte (CD2AP and Podocin) in DN rats were investigated after the oral administration of EZF. The concentrations of specnuezhenide and wedelolactone in rat kidney were 7.19 and $0.057 \mathrm{mg} / \mathrm{kg}$, respectively. The $\mathrm{T}_{\max }$ of specnuezhenide and wedelolactone were 2.0 and $1.50 \mathrm{~h}$, respectively. Their $\mathrm{C}_{\max }$ were, respectively, $30.24 \pm 2.68$ and $6.39 \pm 0.05 \mu \mathrm{g} / \mathrm{L}$. Their $\mathrm{AUC}_{(0-\infty)}$ were $123.30 \pm 2.68$ and $16.56 \pm 0.98 \mu \mathrm{g} / \mathrm{L} * \mathrm{~h}$, respectively. Compared with the model group, the blood glucose and the 24-hour urinary protein were significantly decreased $(\mathrm{P}<0.05)$ after 16 weeks' treatment of EZF. The expressions of Podocin and CD2AP protein/mRNA were increased $(\mathrm{P}<0.05)$. The deteriorate of glomerular morphology was alleviated under the treatment of EZF. EZF prominently decreased the levels of inflammatory factors $(\mathrm{P}<0.05)$. MDA was significantly decreased $(\mathrm{P}<0.05)$ with the significant increase of SOD activity $(\mathrm{P}<$ 0.05 ) in EZF groups. All the results proved that EZF repaired glomerular mesangial matrix, protected renal tubule, and improved renal function in DN rats by upregulating the expression of Podocin and CD2AP protein/mRNA in podocytes.
\end{abstract}

\section{Introduction}

In recent years, effective monomers or extracts from natural plants have been widely applied to the treatment of diabetic nephropathy (DN) [1-3]. Erzhi formula was composed of Ligustri lucidi fructus and Eclipte herba by the ratio 1:1 [4]. It can provide nutrients to the liver and kidneys and strengthen the waist and knee joints [5]. Erzhi formula extract (EZF) contained lots of active compounds such as specnuezhenide, wedelolactone, oleanolic acid, acetyl oleanolic acid, and ursolic acid $[6,7]$. Among these compounds, oleanolic acid ameliorated $\mathrm{DN}$ and protected renal microstructural by reducing oxidative stress and endoplasmic reticulum stress in type II diabetic rats [8-11]. Ursolic acid prevented renal damage by lowering blood glucose, inhibiting the early damage of $\mathrm{DN}$, suppressing oxidation and inflammatory reactions [1214]. The specnuezhenide and wedelolactone resisted DN by inhibiting the signaling pathways related to inflammation such as HIF-1 $\alpha /$ VEGF [15] and NF-kappa B [16].

$\mathrm{DN}$ was one of the major microvascular complications of diabetes [17]. It was characterized by accumulating glomerular extracellular matrix and tubulointerstitial compartments, thickening the intrarenal vasculature [18-20]. In recent years, the podocytes, glomerular filtration barrier structures [21], have been widely concerned. Podocytes were highly specialized cells of the renal glomerulus that wrapped around capillaries and neighbor cells of the Bowman's capsule [2225]. Podocytes injury was extremely important in the pathogenesis of DN and was also the important target of many pathogenic factors in the process of DN [26]. When the podocytes damage was exceeded, the compensatory capacity, the filter membrane, could be destroyed which induced in large amounts of proteinuria [27-30]. Proteinuria was an important clinical marker of early DN. Therefore, the 
protection of podocytes was closely related to the treatment of DN. Many effective compounds and herb extracts have been discovered to protect or repair podocyte injury, such as Cordyceps sinensis, tripterygium wilfordii polyglycosidium [31], and astragalus membranaceus [32]. CD2AP and Podocin were important structural proteins of the podocyte silt membrane and maintained the glomerular filtration function and regulated proteinuria $[33,34]$.

The development of DN was directly linked to inflammation [35]. There were many inflammatory factors, such as IL-17A [36], IL- $\beta$, TNF- $\alpha$, IL-6, MCP-1, ICAM-1, VCAM1 , IL-1, and IL-18 [37]. However, IL- $\beta$, TNF- $\alpha$, and IL-6 were classical inflammatory factors for studying DN [38]. Superoxide dismutase (SOD) was an extremely important antioxidant enzyme in the human body that had multiple functions such as antiaging, immune regulation, and lipid regulation. Malondialdehyde (MDA) levels usually reflected the oxidative damage in cells and tissues [39]. In summary, these indicators were important for studying DN.

In this paper, a HPLC-MS/MS method for simultaneously detecting specnuezhenide and wedelolactone was established which was applied to the quality control and the pharmacokinetic study of EZF. And then, by replicating the DN in rats, the effects of EZF on blood glucose, proteinuria, renal index, pathological changes of renal tissue, expression of Podocin/CD2AP protein and mRNA, the IL- $\beta$, TNF- $\alpha$, IL6 , and MDA levels, and SOD activity in renal tissue were evaluated. Finally, we described the mechanism of EZF in treating and reversing the DN.

\section{Materials and Methods}

2.1. Instruments and Reagents. Ecliptae Herbs and Ligustri Lucidi Fructus were purchased from Zhenjiang Ren Tang Pharmaceutical Co., Ltd. (Zhenjiang, China) and identified by Professor Chen Jun of Jiangsu University as Eclipta prostrata L. and Ligustrum lucidum Ait., respectively. Streptozotocin was obtained from Sigma-Aldrich (S0131, Sigma). Irbesartan was purchased from Sanofi Winthrop Industries (0.15 g/tablet, H20040494, Beijing). Glucose meter was Ouch surestep lood (ONETOUCH, Johnson China Co., Ltd.). Three triple quadrupole LC-MS/MS (Thermo TSQ Quantum) was purchased from Thermo Fisher Scientific (America) which is equipped with QED-MS/MS system. The polyclonal antibody of rabbit anti-rat Podocin was purchased from American Abcam Company (GR211434-1, USA). The polyclonal antibody of rabbit CD2AP was provided by Beijing Boosen biological (G3828, Bioss Antibodies, China); TRIzol Reagent was provided by Life Technologies (50175111, Life Technologies, USA); M-MLV reverse transcription kit was obtained from American Invitrogen Company (RP1105, Invitrogen Co., Ltd., USA). Primers were purchased from Suzhou Genewiz Technology Co., Ltd. (NTKW-20171103, Suzhou, China). Highsugar and high-fat feed were provided by Beijing Botai Hongda Biological Technology Co., Ltd. (S20160808, Beijing, China). The first strand of cDNA Synthetic kit was purchased from Vazyme Biotech Co., Ltd. (04896866001, Nanjing, China). Ethanol, chloroform, and isopropanol were analytical pure grade reagents, obtained from Sinopharm Group Co.,
Ltd. IL-1 $\beta$ (A1001), IL-6 (A1005), TNF- $\alpha$ (A1012), Superoxide Dismutase (SOD, A001-3), Malonaldehyde (MDA, A003-1), and urine protein assay kit (C035-2) were purchased from Nanjing built Biotechnology Co., Ltd (Nanjing, China).

2.2. Animals. Male SPF SD rats weighing $220 \pm 20$ g were originally obtained from the Animal Center of Jiangsu University. The animal certificate number was SYXK 20130036. The animal experiments were conducted by the Animal Ethics Committee. The rats were in-house fed separately at $23^{\circ} \mathrm{C}$ and $40 \%$ humidity. Animals were maintained on standard laboratory chow and daily 12-hour light/dark cycles. All animals were provided with standard ordinary feed and water.

2.3. Preparation of EZF. The dried Erzhi formula was crushed and extracted with $80 \%$ aqueous ethanol for two times (2 $\mathrm{h}$ for each) under heat and reflux. The extracts were dried by rotary evaporator after two extracts were combined and the ethanol was recovered. The dry powders $(0.2 \mathrm{~g} / \mathrm{g}$ of crude drug) were kept dry and preserved in dark place for subsequent experiments.

\subsection{Simultaneous Determination of Main Components in EZF}

2.4.1. Chromatographic Conditions. The chromatographic separations were performed on Agilent ZORBAX SB- $\mathrm{C}_{18}$ column $(150 \mathrm{~mm} \times 2.1 \mathrm{~mm}, 5 \mu \mathrm{m})$. The HPLC mobile phase which consisted of methanol (A) and $0.1 \%$ formic acid (B) was used to conduct gradient elution. The flow rate of gradient elution was $0.3 \mathrm{~mL} / \mathrm{min}$, and the column temperature was room temperature.

2.4.2. Mass Spectrometry Conditions. The mass spectrometry analysis was performed with an electrospray ionization source in the negative ion detection mode, and the scanning mode was multiple reaction monitoring. Resolution was unit mass resolution. Capillary voltage and temperature were 3000 $\mathrm{V}$ and $350^{\circ} \mathrm{C}$, respectively. Nitrogen was used as collision gas. The mass spectral parameters of the two compounds were shown in Table 1.

2.5. Solutions and Samples Preparation. EZF samples: 20 mg of specnuezhenide and wedelolactone were precisely weighed, respectively, and then appropriate amounts of methanol were added for ultrasonic-assisted dissolution. Then it was set to $100 \mathrm{~mL}$ as standard solution. The standard solution was diluted into a series of different concentrations for the establishment of standard curve. $10 \mathrm{mg}$ of EZF was weighed and dissolved with $10 \mathrm{~mL}$ methanol by the assistant of ultrasound and then fixed up to $50 \mathrm{~mL}$ by methanol. The microporous filter membrane with $0.22 \mu \mathrm{m}$ was used for filtration for subsequent HPLC-MS/MS detection. All samples were operated in parallel 6 times.

Plasma sample $(n=6)$ : EZF was suspended in $0.5 \%$ sodium carboxymethyl cellulose and administrated orally. 0.4 $\mathrm{mL}$ rat blood was collected and placed in a centrifuge tube with heparin at $0.00,0.083,0.25,0.5,1,1.5,2,4,6,8,10$, and 12 $\mathrm{h}$ from posterior orbital plexus, respectively. All the samples were centrifuged at $5000 \mathrm{r} / \mathrm{min}$ for $15 \mathrm{~min}$. And then, $0.1 \mathrm{~mL}$ 
TABLE 1: The mass spectral parameters of specnuezhenide and wedelolactone.

\begin{tabular}{|c|c|c|c|c|c|}
\hline Chemical compound & Parent ion $(\mathrm{m} / \mathrm{z})$ & daughter ion $(\mathrm{m} / \mathrm{z})$ & Residing time (ms) & Cracking voltage (V) & Collision energy $(\mathrm{V})$ \\
\hline \multirow{2}{*}{ Wedelolactone } & \multirow{2}{*}{313} & $298.1^{*}$ & 100 & 81 & 23 \\
\hline & & 186.0 & 100 & 81 & 38 \\
\hline \multirow{2}{*}{ Specnuezhenide } & \multirow{2}{*}{685.21} & $523.3^{*}$ & 100 & 89 & 21 \\
\hline & & 299.1 & 100 & 89 & 27 \\
\hline
\end{tabular}

${ }^{*}$ Quantitative ion.

supernatant was taken out and $0.2 \mathrm{~mL}$ acetonitrile was added to precipitate protein.

Tissue sample $(n=6)$ : Rats were orally administrated with EZF (10 g/kg). 2 hours later, their heart, liver, spleen, lung, and kidney were taken out, washed clean, and homogenated $(1.0 \mathrm{~g} / \mathrm{mL})$. $0.2 \mathrm{~mL}$ supernatant from homogenate liquid was taken out and $0.2 \mathrm{~mL}$ acetonitrile was added to precipitate protein.

All plasma and tissue samples were vortex $(2 \mathrm{~min})$, centrifuged $(10000 \mathrm{r} / \mathrm{min}, 10 \mathrm{~min})$, and filtered $(0.22 \mu \mathrm{m})$ in turn followed by $\mathrm{N}_{2}$ blow dry. The residue was accurately added to $200 \mu \mathrm{L}$ methanol, vortexed $(2 \mathrm{~min})$, centrifuged $(12000 \mathrm{r} / \mathrm{min}, 10 \mathrm{~min})$, and filtered $(0.22 \mu \mathrm{m})$ before HPLCMS/MS analysis.

2.6. Establishment of Diabetic Rat Model and Grouping. After SD rats were fed for 1 week and adapted to the environment, the detection of urine protein and urine sugar was negative. A total of 48 rats were fed with a small dose of STZ, highglucose, and high-fat diet to duplicate DN rats. All rats were fed with high-glucose and high-fat diet for 4 weeks. The rats were fasted for 12 hours and then injected $1 \%$ STZ (40 mg/kg, $0.1 \mathrm{~mol} / \mathrm{L}$ citrate buffer, and $\mathrm{pH} 4.5$ ) by intraperitoneal injection. The caudal vein blood glucose was measured after $72 \mathrm{~h}$, and rats with persistent hyperglycemia over $16.7 \mathrm{mmol} / \mathrm{L}$ were considered to be diabetic model and selected for further experiments [40, 41]. During the modeling process, the dead rats and the noncompliance rats were excluded. The diabetic rats were randomly divided into model group (MOD), Irbesartan (IRB, $15 \mathrm{mg} / \mathrm{kg}$ ) group [42, 43], EZF high-dose (EZF-H, $15 \mathrm{~g} / \mathrm{kg}$ ) group, EZF middle-dose (EZF-M, 10 g/kg) group, and EZF low-dose (EZF-L, 5 g/kg) group $(n=8)$. Rats in normal group were fed same volume of distilled water, and all groups were continuously fed for 16 weeks.

\subsection{Related Index in the Treatment of DN}

2.7.1. Blood Glucose. After the intervention of the EZF, the blood glucose level of samples was measured in the second, fifth, and eighth weeks, which were taken from the tail veins of rats.

2.7.2. Urine Protein and Inflammation Markers. The total of 24 hours urinary protein and the concentration of IL- $\beta$, IL6 and TNF- $\alpha$ in the renal were detected according to the instructions and steps of kit. Standard solution holes and sample holes were, respectively, set on the test board, and then $50 \mu \mathrm{L}$ standard solution of different concentrations was added into the standard solution. After the sample was added into the sample hole $(10 \mu \mathrm{L})$, the diluent was added (40 $\mu \mathrm{L})$. Standard and sample holes were added with horseradish peroxidase (HRP) labelled antibody $(100 \mu \mathrm{L})$ to detect the antibody. The reaction pore was sealed with the sealing plate membrane and kept in $37^{\circ} \mathrm{C}$ water bath for $60 \mathrm{~min}$. Discard the liquid, dry the plates, fill them up with washing fluid for 1 minutes, shake off the washing liquid, dry the plates, and repeat the washing process 5 times. The substrates were added $(50 \mu \mathrm{L})$ to all the holes and incubated for 15 minutes at $37^{\circ} \mathrm{C}$. 15 minutes later, the OD value of each pore was measured at $450 \mathrm{~nm}$ wavelength. The standard curve was drawn with the standard concentration as abscissa and the OD as the ordinate, and the corresponding concentration of the OD value of the sample was found on the standard curve.

2.7.3. Renal Index. After the rats were sacrificed, the bilateral renal tissues were taken. Then renal tissues were weighed after the renal surface blood was blotted with filter paper.

$$
\begin{aligned}
\text { Renal index }= & \frac{\text { total bilateral renal weight }(\mathrm{g})}{\text { body weight }(\mathrm{kg})} \\
& \times 100 \%
\end{aligned}
$$

2.7.4. Renal Pathological Examination. The renal cortex was taken and fixed by $10 \%$ formaldehyde for 24 hours. It was dehydrated by various concentrations of ethanol $(70 \%, 80 \%$, $90 \%, 95 \%$, and $100 \%$ ) in turn for 30 min each followed by rinsing with water, cleared in xylene and embedded in paraffin and cut into $2 \mu \mathrm{m}$ thick slice. Finally, the pathological changes of renal tissues were observed under light microscope after $\mathrm{HE}$ and PAS staining.

2.7.5. Expression of CD2AP and Podocin Protein/mRNA. The expressions of CD2AP and Podocin protein were detected by Western blotting. After rat renal tissue $60 \mathrm{mg}$ was weighed, RIPA lysis buffer was used to extract RNA and the concentrations of proteins were quantified with Bradford protein quantitation kit. $30 \mu \mathrm{g}$ sample was weight for each group. The solution was well-mixed after the total protein samples and the buffer solution of the protein gel electrophoresis were added to each group sample. The mixtures were ice bathed after denaturation for $10 \mathrm{~min}$ at $95^{\circ} \mathrm{C}$. These samples (30 $\mu \mathrm{g}$ ) were slowly added into the gel hole. When the electrophoresis apparatus is under the stabilivolt state of $80 \mathrm{~V}$, the samples passed through the spacer gel and the separation gel (voltage $8 \mathrm{~V} / \mathrm{cm}$ ). When the dye was electrophoresed to a suitable position in the separation gel, the samples were 
transferred to PVDF membrane on ice. The PVDF membrane was blocked with $5 \%$ skim milk powder at $4^{\circ} \mathrm{C}$. The Podocin primary antibody (1:1000) and the CD2AP primary antibody (1:300) of rabbit anti-rat were added to PVDF membrane. The primary antibody membrane was added to secondary antibody solution (1:5000) after primary antibody membrane was washed with TBST. At room temperature, secondary antibody membrane was shaken slowly in dark. The membrane was washed after 60 minutes, was colored with TMB, was exposed, and was washed film. The gray ratio of the target protein/ $\beta$-actin was used to express the relative ratio of the target protein. Specific operations are shown in the literature [44]. Primer sequences and PCR product sizes are shown in the Table 5 .

2.8. Statistical Processing. Experimental data were expressed as average value \pm standard deviation $(\bar{x} \pm s)$, and onedimensional analysis of variance was performed by using GraphPad Prism 5.0 software. $\mathrm{P}<0.05$ was considered a significant difference. The DAS 2.0 software was applied to calculate the main pharmacokinetic parameters of specnuezhenide and wedelolactone, respectively.

\section{Results}

\subsection{Chemical Composition Analysis}

\subsubsection{Method Validation}

(1) Standard Curve and Linear Range. Linear regression equation was figured through the peak area $\mathrm{Y}$ and concentration $\mathrm{X}$ $(\mu \mathrm{g} / \mathrm{mL})$. The linear regression equations of specnuezhenide and wedelolactone were $\mathrm{Y}=123.408+181.663 \mathrm{X}\left(\mathrm{R}^{2}=0.9999\right)$ and $\mathrm{Y}=92127.2+3822.94 \mathrm{X}\left(\mathrm{R}^{2}=0.9987\right)$. The results showed that there was a good linearity in the range of $0.01-$ $20 \mu \mathrm{g} / \mathrm{mL}$ for specnuezhenide and wedelolactone. Their limit of determination (LOQ) was $0.05 \mu \mathrm{g} / \mathrm{mL}$. The representative HPLC-MS/MS spectrum was shown in Figures 1(a) and 1(b).

(2) Stability and Recovery. The abovementioned standard solution was accurately taken at $0,1,2,4$, and 8 hours and analyzed. The relative standard deviations of specnuezhenide and wedelolactone peak areas were $0.13 \%$ and $1.42 \%$, respectively, indicating that these compounds had good stability. In addition, the known contents of 3 samples were taken and 0.5 times of standard solution were accurately added to these sample solutions. According to the preparation method of the samples, the recovery rates were calculated. The results showed that the recoveries of specnuezhenide and wedelolactone were $101.2 \%$ and $100.3 \%$, indicating that they had good recovery rates.

$10 \mu \mathrm{L}$ mixed standard solution $(10 \mu \mathrm{g} / \mathrm{mL})$ was added into $0.1 \mathrm{~mL}$ blank plasma samples which were obtained from rat and their peak areas were measured by HPLC-MS/MS as "A" after the pretreatment. Another $10 \mu \mathrm{L}$ mixed standard solution $(10 \mu \mathrm{g} / \mathrm{mL})$ was added into $0.1 \mathrm{~mL}$ methanol with the same operation according to the plasma samples and the peak areas were measured as "B." And then calculate their recovery by " $(A / B) \times 100 \%$ ". The recovery rate of specnuezhenide was
TABLE 2: The distribution of specnuezhenide and wedelolactone in rat tissues $(\overline{\mathrm{x}}, \mathrm{n}=6)$.

\begin{tabular}{lcc}
\hline Tissues & \multicolumn{2}{c}{ Components $(\mathbf{m g} / \mathbf{k g})$} \\
& Specnuezhenide & Wedelolactone \\
\hline Spleen & $/$ & 5.78 \\
Lung & 8.56 & 0.089 \\
Heart & $/$ & $/$ \\
Liver & 1.52 & 0.20 \\
Renal & 7.19 & 0.057 \\
\hline
\end{tabular}

$84.36 \%$ and the recovery rate of wedelolactone was $86.06 \%$, which indicated that they all had good recovery rates.

(3) The Content of specnuezhenide and Wedelolactone in EZF. The content of specnuezhenide in EZF was $9.79 \%$, and the content of wedelolactone was $0.61 \%$. The representative HPLC-MS/MS spectrum was shown in Figures 1(a) and 1(b).

\subsection{The Tissue Distribution and Pharmacokinetics of EZF in Rats}

3.2.1. The Tissue Distribution of Specnuezhenide and Wedelolactone. As shown in Table 2, the concentrations of specnuezhenide in the lung, renal, and liver tissues were 8.56, 7.19 , and $1.52 \mathrm{mg} / \mathrm{kg}$, respectively, after the oral administration of EZF, but the specnuezhenide in heart and spleen was not detected. The concentrations of wedelolactone in spleen, lung, liver, and renal were $5.78,0.089,0.057$, and $0.20 \mathrm{mg} / \mathrm{kg}$, respectively, but the wedelolactone in heart was not detected. Therefore, specnuezhenide was easily distributed in the lungs and kidney and wedelolactone was easily distributed in the spleen after the administration of EZF. The representative HPLC-MS/MS spectrum was shown in Figure 1(c).

3.2.2. The Pharmacokinetics Study of EZF in Rats. According to the pharmacokinetics parameters, the peak time $\left(\mathrm{T}_{\max }\right)$ of specnuezhenide and wedelolactone was $2.0 \mathrm{~h}$ and 1.50 $\mathrm{h}$, respectively, indicating that the absorption of wedelolactone was faster than specnuezhenide. However, the $\mathrm{C}_{\max }$ of specnuezhenide $(30.24 \pm 1.65 \mu \mathrm{g} / \mathrm{L})$ was bigger than wedelolactone $(6.39 \pm 0.05 \mu \mathrm{g} / \mathrm{L})$. In addition, the $\mathrm{AUC}_{(0-\infty)}$ of specnuezhenide $(123.30 \pm 2.68 \mu \mathrm{g} / \mathrm{L} * \mathrm{~h})$ was also significantly higher than wedelolactone $(16.56 \pm 0.98 \mu \mathrm{g} / \mathrm{L} * \mathrm{~h})$. The results were shown in Figure 2 and Table 3.

3.3. Hypoglycemic Effect of EZF. In the normal group, the blood glucose level was maintained at $4.49 \pm 0.02 \mathrm{mmol} / \mathrm{L}$. In model, the blood glucose level gradually tends to be stable $(23.78 \pm 0.21 \mathrm{mmol} / \mathrm{L})$ after 4 weeks $(\mathrm{P}<0.01)$. After 16 weeks' treatment of Irbesartan, the blood glucose level reached $5.10 \pm 0.20 \mathrm{mmol} / \mathrm{L}$, which was not significantly different compared with the normal group. The blood glucose level did not decrease significantly after 4 weeks' treatment of EZF. However, the blood glucose level in DN rats decreased significantly from $8^{\text {th }}$ to $16^{\text {th }}$ week. Compared with the model, the blood glucose levels of EZF-H $(15.43 \pm 0.39 \mathrm{mmol} / \mathrm{L})$, 


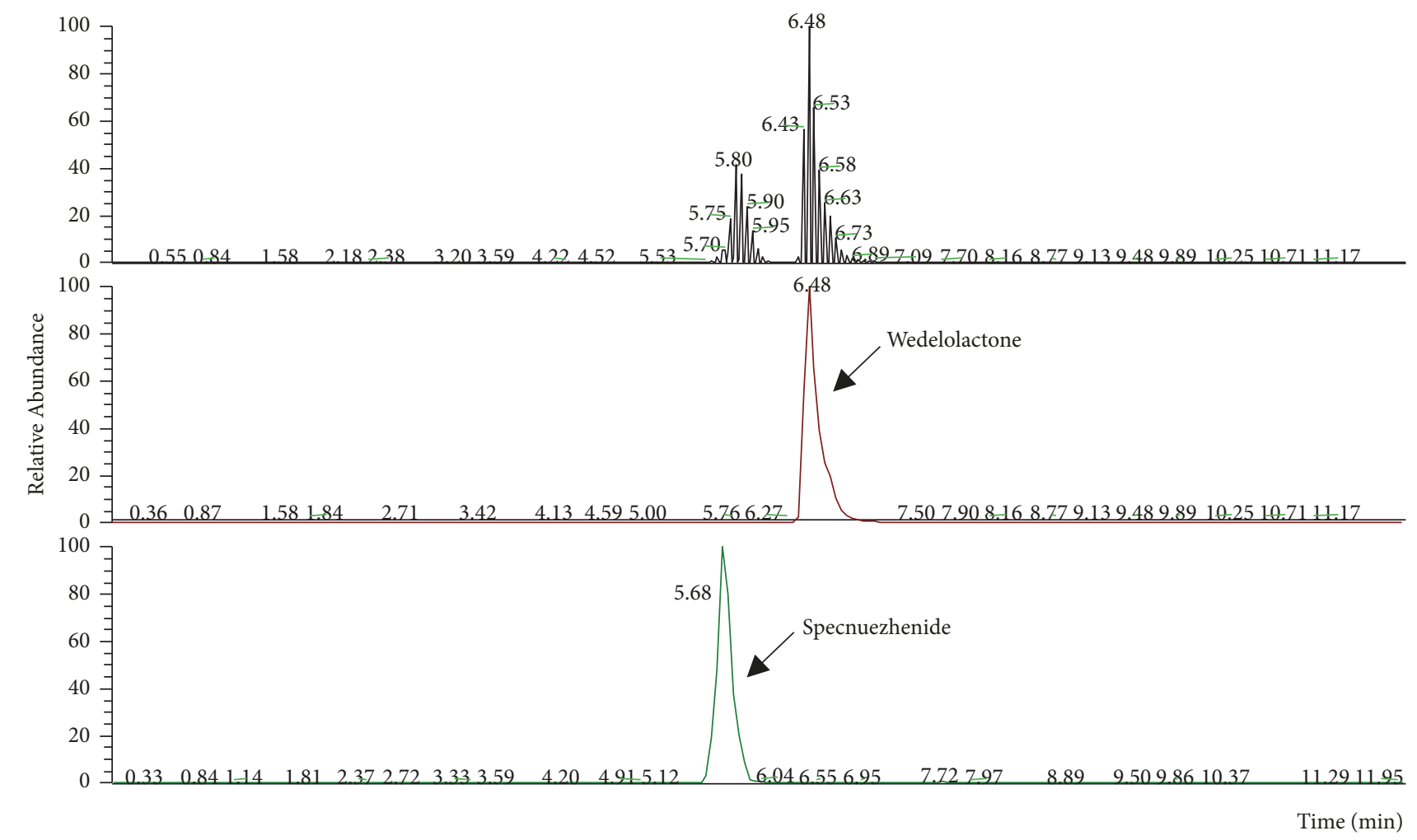

(a)

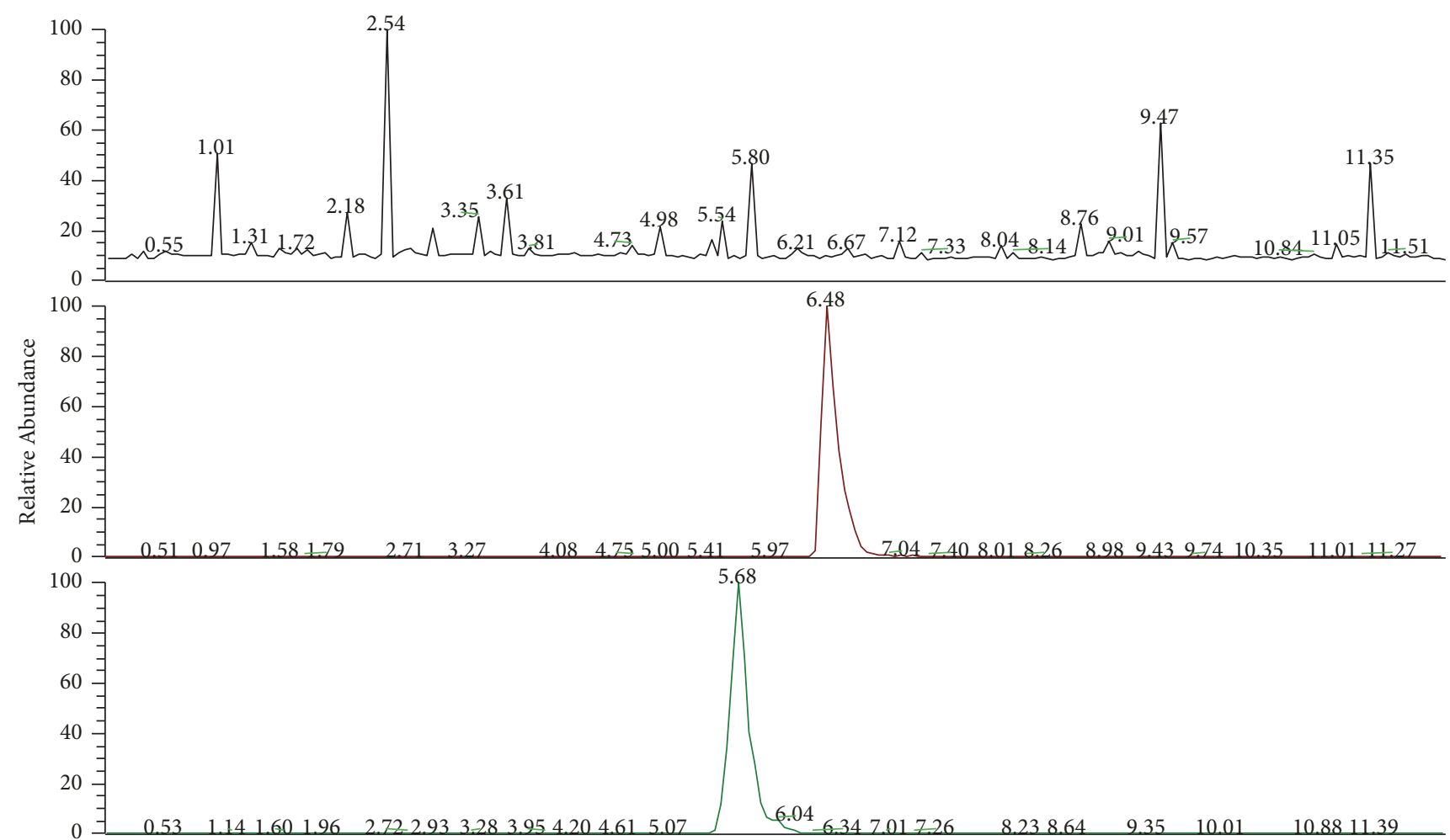

Time (min)

(b)

Figure 1: Continued. 


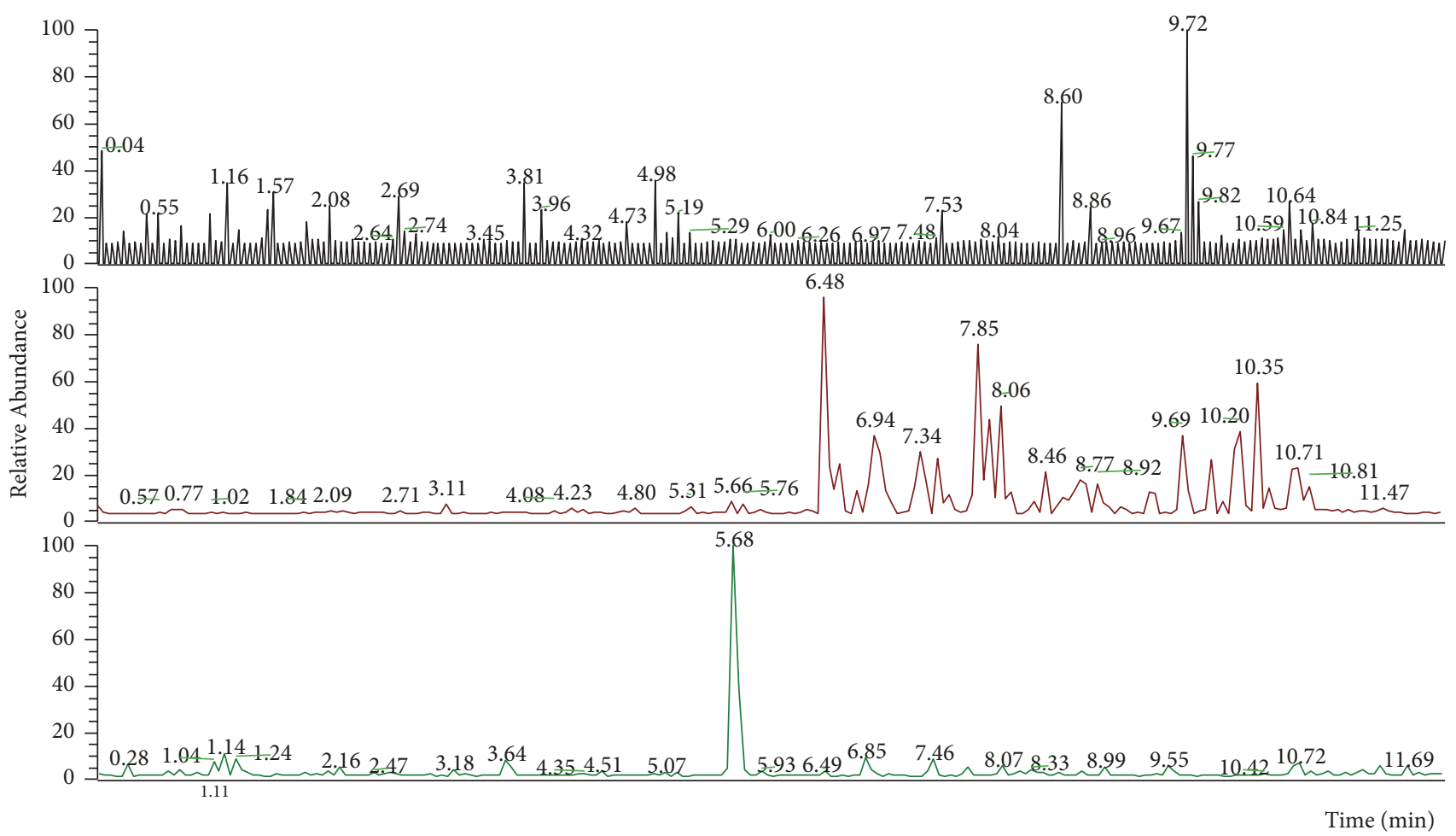

(c)

FIgURE 1: HPLC-MS/MS chromatogram of specnuezhenide and wedelolactone in EZF, serum and tissue. (a) The HPLC-MS/MS chromatogram of mixed standard; (b) the HPLC-MS/MS chromatogram of EZF; (c) the HPLC-MS/MS chromatogram in serum and tissue after oral administration of EZF.

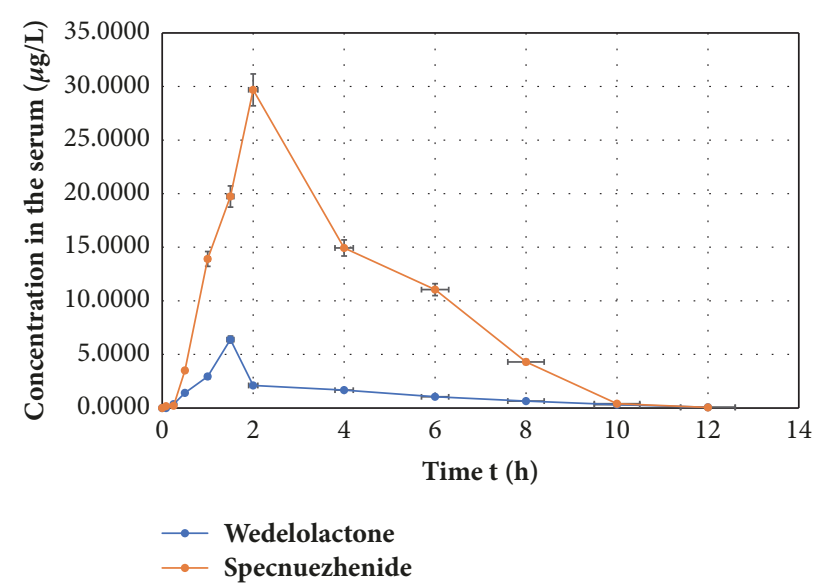

FIGURE 2: The concentration-time curve of specnuezhenide and wedelolactone in rats after oral administration of $\operatorname{EZF}(\bar{x}, \mathrm{n}=6)$.

EZF-M (18.47 $\pm 0.15 \mathrm{mmol} / \mathrm{L})$, and EZF-L $(20.67 \pm 0.49$ $\mathrm{mmol} / \mathrm{L})$ were significantly decreased $(\mathrm{P}<0.05)$ at the $16^{\text {th }}$ week. Different doses of EZF group showed dose dependence (Figure 3).

3.4. The Effect of Reducing Proteinuria. Compared with normal group, the $24 \mathrm{~h}$ urinary protein level in the model group was increased significantly $(\mathrm{P}<0.01)$. Compared with the model group, 24h urinary protein of Irbesartan group was decreased significantly $(19.60 \pm 0.24 \mathrm{mg}, \mathrm{P}<0.05)$. The $24 \mathrm{~h}$ urinary protein levels in EZF-H (20.72 $\pm 0.45 \mathrm{mg})$, EZF$\mathrm{M}(24.88 \pm 0.42 \mathrm{mg})$, and EZF-L $(28.65 \pm 0.46 \mathrm{mg})$ were significantly reduced $(\mathrm{P}<0.05)$. The results were shown in Figure 4.

3.5. Body Weight, Renal Weight, and Renal Index Changes. Compared with the normal group, the body weight of model group was decreased significantly $(\mathrm{P}<0.01)$; renal weight and renal index increased significantly $(\mathrm{P}<0.01)$. Compared with the model group, Irbesartan significantly reversed the body weight and renal weight of the model group $(\mathrm{P}<$ $0.01)$ and the renal index decreased significantly $(P<0.01)$. After the intervention of the EZF, EZF-H and EZF-M groups significantly reversed the body weight, kidney weight, and renal index of diabetic rats $(\mathrm{P}<0.05)$. The specific results are shown in Table 4.

3.6. Inflammatory Factors and Antioxidant Markers in the Renal Injury. Compared with the normal group, IL- $\beta$, TNF$\alpha$ and IL- 6 in the renal podocytes of model were significantly increased $(\mathrm{P}<0.01)$. The Irbesartan decreased these inflammatory factors in the model group $(\mathrm{P}<0.01)$. Compared with the model group, the EZF-H and EZF-M significantly reduced IL- $\beta$, TNF- $\alpha$, and IL- $6(\mathrm{P}<0.05)$. The results are shown in Figure 5. 
TABLE 3: The specnuezhenide and wedelolactone pharmacokinetic parameters of EZF in rats by intragastric administration $(\bar{x} \pm$ SD, $\mathrm{n}=6)$.

\begin{tabular}{lccc}
\hline Statistical moment parameter & Unit & Wedelolactone & Specnuezhenide \\
\hline AUC $(0-\mathrm{t})$ & $\mu \mathrm{g} / \mathrm{L} * \mathrm{~h}$ & $13.77 \pm 1.17$ & $116.60 \pm 14.15$ \\
AUC $(0-\infty)$ & $\mu \mathrm{g} / \mathrm{L} * \mathrm{~h}$ & $16.56 \pm 0.98$ & $123.30 \pm 2.68$ \\
AUMC $(0-\mathrm{t})$ & & $51.09 \pm 2.89$ & $419.01 \pm 9.19$ \\
AUMC $(0-\infty)$ & & $69.13 \pm 5.51$ & $437.67 \pm 13.92$ \\
MRT $(0-\mathrm{t})$ & $\mathrm{h}$ & $3.12 \pm 0.35$ & $3.63 \pm 0.08$ \\
MRT $(0-\infty)$ & $\mathrm{h}$ & $4.52 \pm 0.43$ & $3.52 \pm 0.22$ \\
VRT $(0-\mathrm{t})$ & $\mathrm{h} \wedge 2$ & $5.77 \pm 0.42$ & $4.42 \pm 0.05$ \\
VRT $(0-\infty)$ & $\mathrm{h} \wedge 2$ & $14.48 \pm 1.01$ & $5.55 \pm 0.38$ \\
$\mathrm{t} 1 / 2 \mathrm{z}$ & $\mathrm{h}$ & $2.95 \pm 0.40$ & $2.36 \pm 0.28$ \\
$\mathrm{~T}_{\max }$ & $\mathrm{h}$ & $1.50 \pm 0.00$ & $2.00 \pm 0.00$ \\
$\mathrm{C}_{\max }$ & $\mu \mathrm{g} / \mathrm{L}$ & $6.39 \pm 0.05$ & $30.24 \pm 1.65$ \\
\hline
\end{tabular}

TABLE 4: Effect of EZF on body weight, renal weight, and renal index in diabetic rats $(\bar{x} \pm S D, n=8)$.

\begin{tabular}{lccc}
\hline Groups & Body weight $(\mathrm{g})$ & renal weight $(\mathrm{g})$ & renal Index $(\times 100)$ \\
\hline Normal & $710.32 \pm 11.54$ & $1.57 \pm 0.04$ & $0.22 \pm 0.004$ \\
Model & $327.83 \pm 4.83^{*}$ & $1.74 \pm 0.05^{\#}$ & $0.53 \pm 0.020^{\#}$ \\
Irbesartan & $376.37 \pm 6.77^{* *}$ & $1.58 \pm 0.04^{* *}$ & $0.42 \pm 0.013^{* *}$ \\
EZF-H & $361.63 \pm 4.30^{* *}$ & $1.59 \pm 0.05^{* *}$ & $0.44 \pm 0.019^{* *}$ \\
EZF-M & $345.70 \pm 3.46^{*}$ & $1.65 \pm 0.02^{*}$ & $0.48 \pm 0.004^{* *}$ \\
EZF-L & $334.60 \pm 1.59$ & $1.68 \pm 0.05$ & $0.50 \pm 0.012$ \\
\hline
\end{tabular}

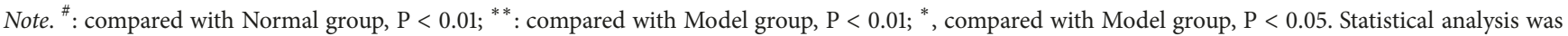
completed by GraphPad Prism 5.0 software.

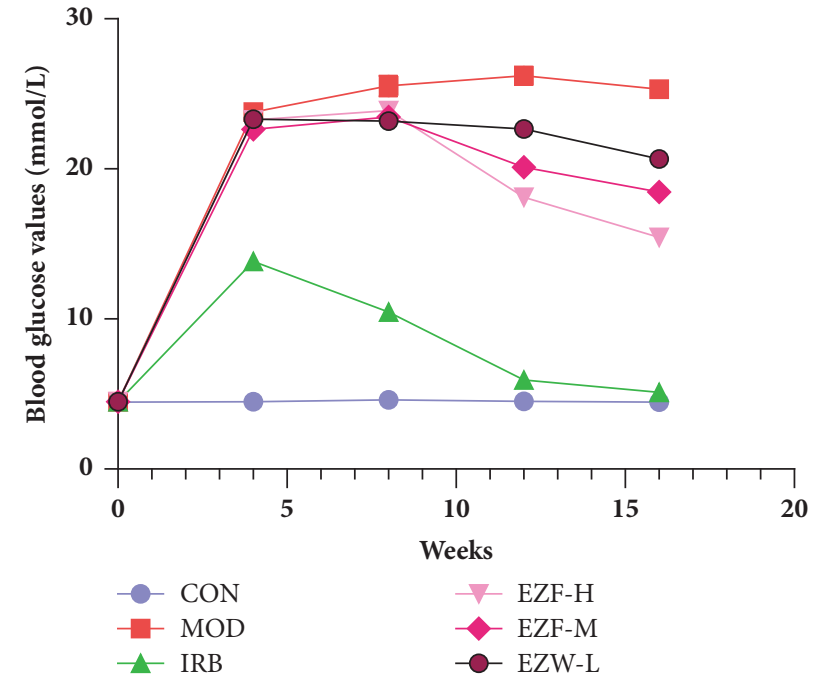

Figure 3: The effect of EZF on blood glucose at different time in diabetic rats $(\bar{x}, \mathrm{n}=8)$.

The results of antioxidant assays showed that SOD activity in the renal of the model group decreased significantly $(\mathrm{P}<$ $0.01)$ and the MDA concentration increased significantly $(\mathrm{P}<$ 0.01). Compared with the model group, the MDA in rats renal were significantly reduced under the treatment of EZF-H and EZF-M $(\mathrm{P}<0.05)$. Furthermore, all EZF groups significantly increased SOD activity $(\mathrm{p}<0.05)$. The results are shown in Figure 6.

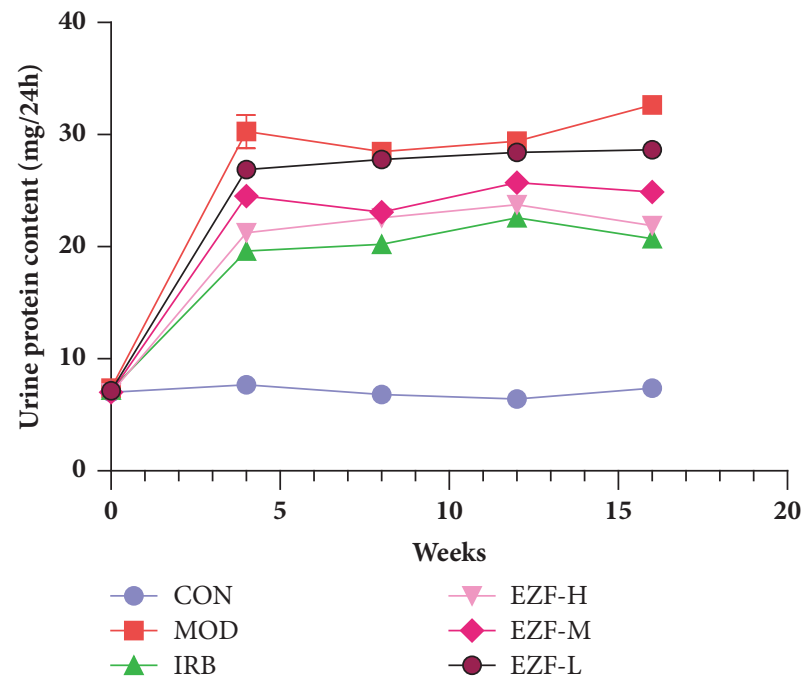

FIgURE 4: The effect of EZF on 24 hours urine protein in diabetic rats $(\bar{x}, \mathrm{n}=8)$.

3.7. HE and PAS Staining. In the Normal group, the kidney had normal renal morphology, clear structure, regular globules, no expansion of capillary lumens, tight arrangement of tubules, normal renal tubular epithelial cell morphology, no glomerular atrophy or hypertrophy, no thickening of the basement membrane, and no mesangial proliferation. Compared with the normal group, the glomerular volume 
TABLE 5: List of primer sequences and PCR product sizes.

\begin{tabular}{lcc}
\hline Name & Sequence & Length \\
\hline podocin-F_Rat & GGTTCTGCATAAAGGTTGTTCAAGA & 169 \\
podocin-R_Rat & TCATGGAAAGGTATTTCCAAGGTCT \\
CD2AP-F_Rat & AGCTTCCTCAGAGAACTTGTTACAT \\
CD2AP-R_Rat & GAAAGAGATGGCTTTGAAGAGTAGC \\
R_GAPDH_F266 & GTGCTGAGTATGTCGTGGAGTC \\
R_GAPDH_R440 & TTGCTGACAATCTTGAGGGA & 204 \\
\hline
\end{tabular}
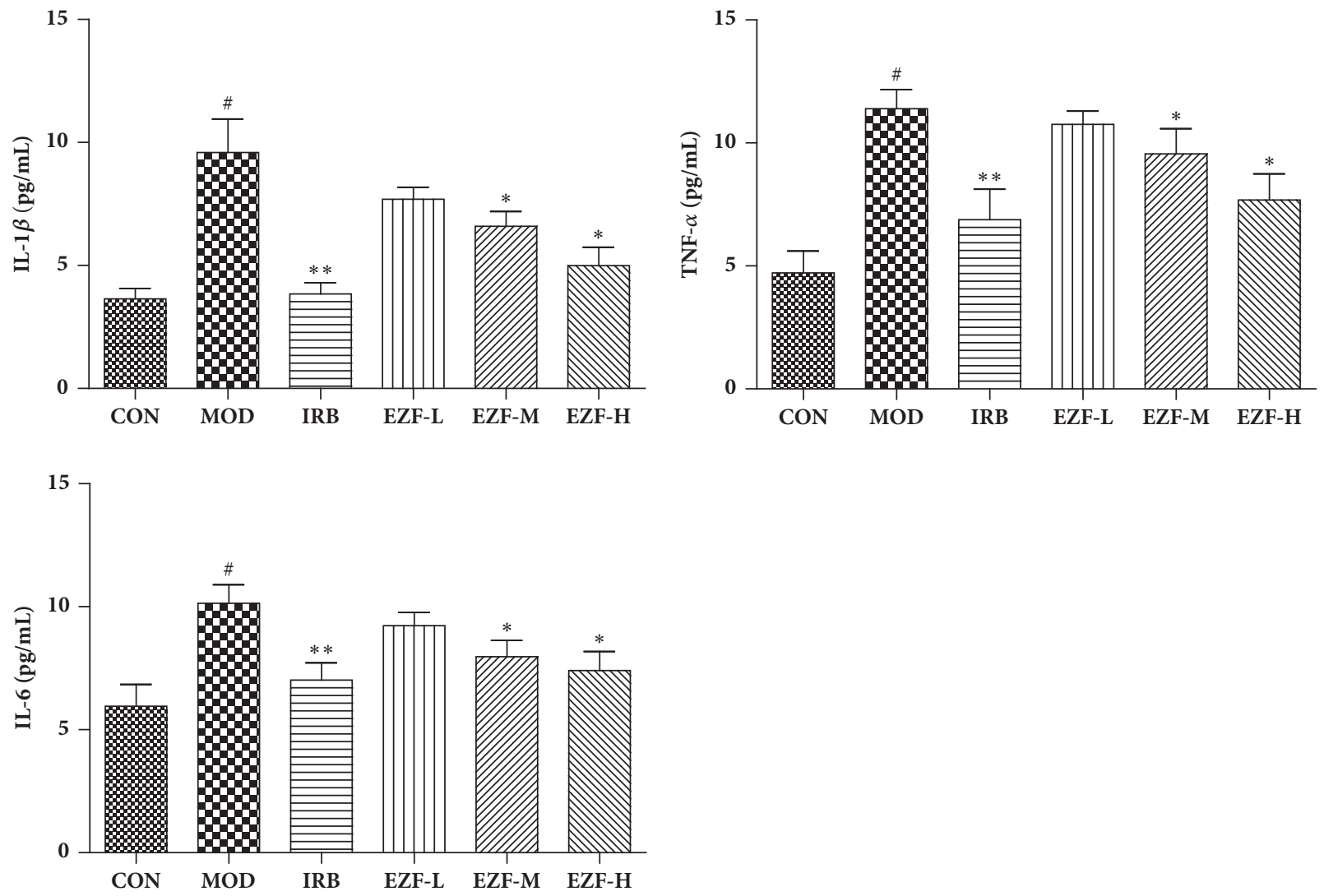

FIGURE 5: The effect of EZF on renal inflammation markers in diabetic rats $(\bar{x}, \mathbf{n}=\mathbf{8})$. CON: blank control group. MOD: model group. IRB: Irbesartan group. EZF-L: Erzhi formula low-dose group. EZF-M: Erzhi formula middle-dose group. EZF-H: Erzhi formula high-dose group. Note. \#: compared with normal group, $\mathrm{P}<0.01$. * * compared with model group, $\mathrm{P}<0.01$. $*$ : compared with model group, $\mathrm{P}<0.05$. Statistical analysis was completed by GraphPad Prism 5.0 software.

of the model group rats increased and the capillary basement membrane thickened significantly. The proliferation of mesangial matrix, vacuolar degeneration of renal tubular epithelial cells, and protein tube type can be observed in the model group. EZF and the Irbesartan groups alleviated the pathological changes of DN rats. The glomerular morphology was basically normal. The thickened basement membrane and the hyperplasia of mild mesangial were obviously observed. There was a small amount of vacuolar degeneration in renal tubular epithelial cells and the lesion extent was significantly lighter than the model group. The HE and PAS staining, the fraction of mesangial matrix area, and renal tubule injury score were shown in Figure 7 (Figures 7(a) and $7(b))$.
3.8. CD2AP and Podocin Protein/Gene Expression. Compared with the normal group, the Podocin and CD2AP protein/gene in the renal tissue of the model group were decreased $(\mathrm{P}<0.01)$. Compared with the model group, the expression of Podocin and CD2AP protein/gene in the IRB, three EZF groups, were increased $(P<0.05)$. The protein and gene expression of Podocin and CD2AP were shown in Figures 8(a) and 8(b), respectively.

\section{Discussion}

So far, HPLC was mainly applied for the quality control of Erzhi formula. Although this method satisfied the determination of the main components in medicinal materials, its 

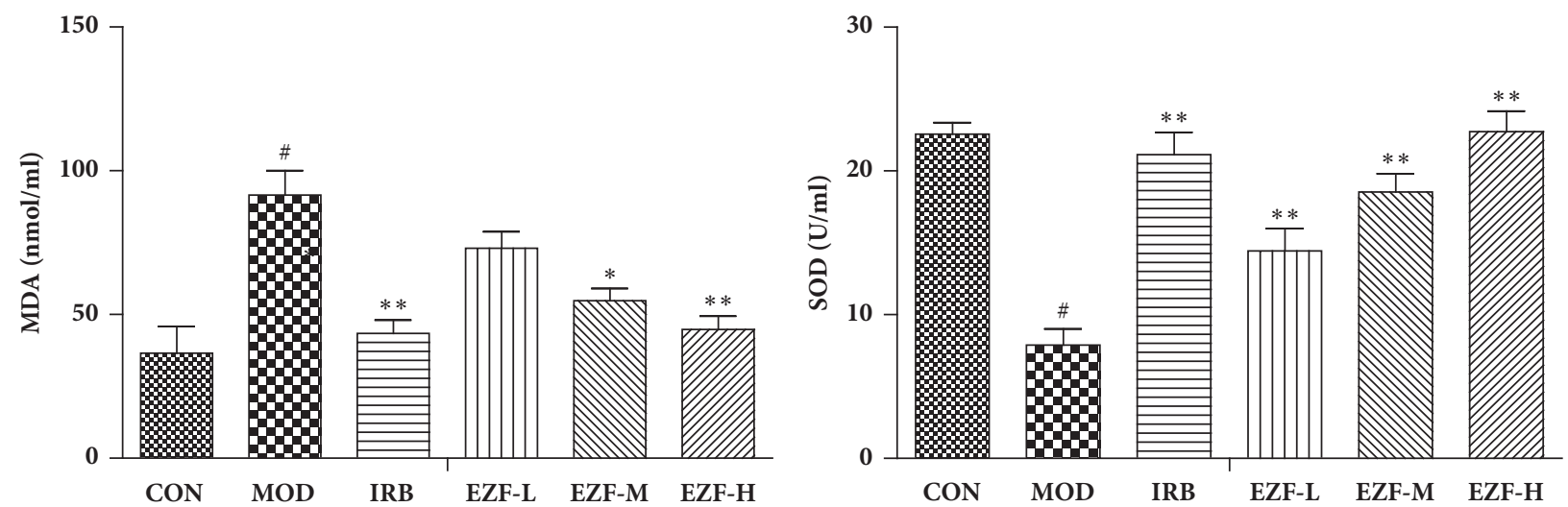

FIGURE 6: SOD activity and MDA concentration in renal of each group $(\bar{x}, \mathbf{n}=\mathbf{8})$. Note. \#: compared with normal group, P $<0.01$. $* *$ : compared with model group, $\mathrm{P}<0.01$ * : compared with model group, $\mathrm{P}<0.05$. Statistical analysis was completed by GraphPad Prism 5.0 software.

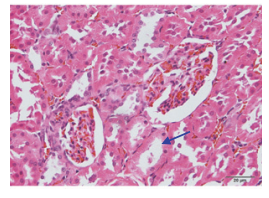

$\mathrm{CON}$

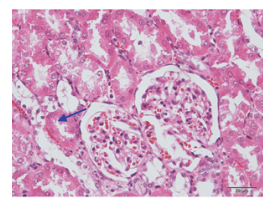

EZF-L

$\longleftarrow$ Renal tubule

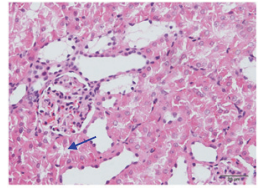

MOD

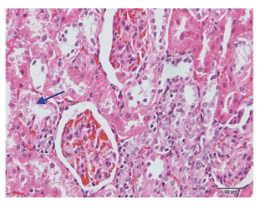

EZF-M

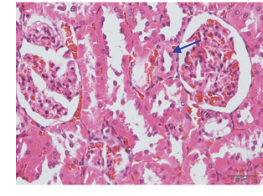

IRB

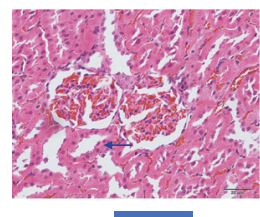

EZF-H

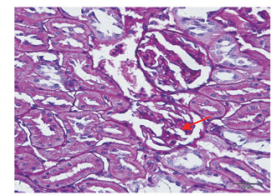

CON

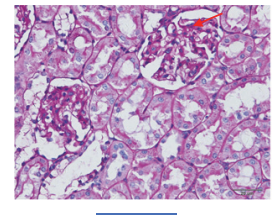

EZF-L

$\leftarrow$ Mesangial matrix

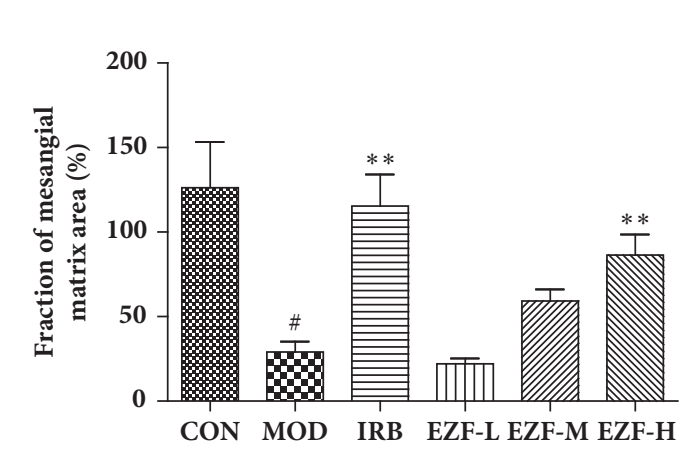

(b)

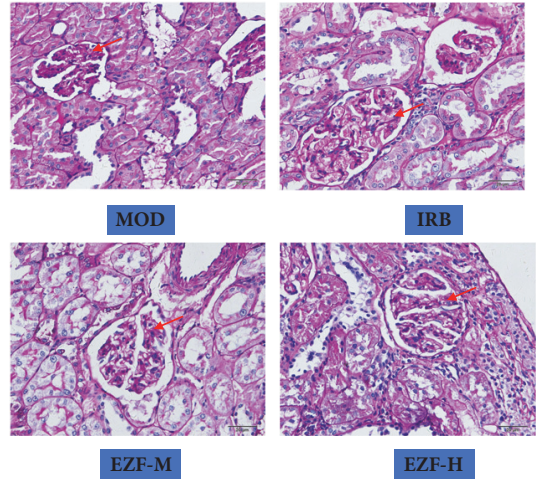

EZF-H

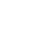 \\ .}

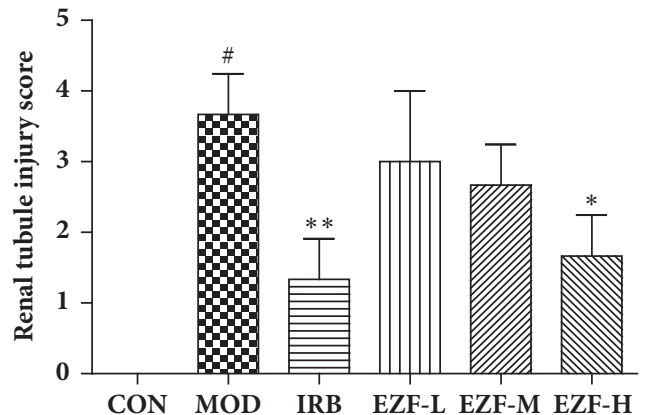

(a)

FIGURE 7: HE and PAS staining of renal pathological changes in rats. (a) HE staining of renal tubule pathological changes $(\times 400)$. (b) PAS staining of glomerular mesangial matrix pathological changes $(\times 400)$. The fraction of mesangial matrix area $=($ mesangial matrix area/glomerular area) $\times 100 \%$. The rules of renal tubule injury score were as follows: 0 points, normal kidney; 1 points, minimal necrosis ( $<5 \%$ renal tubular necrosis); 2 points, mild necrosis (5\%-25\% renal tubular necrosis); 3 points, moderate necrosis (25\%-75\% renal tubular necrosis); 4 points, severe necrosis. Note. \#: compared with normal group, $\mathrm{P}<0.01$. $* *$ : compared with model group, $\mathrm{P}<0.01$. $*$ : compared with model group, $\mathrm{P}<0.05$. Statistical analysis was completed by GraphPad Prism 5.0 software.

sensitivity and accuracy cannot satisfy the needs of biological samples such as serum and tissues. Therefore, the HPLCMS/MS method was established for the first time in simultaneous determination of wedelolactone and specnuezhenide which derived from Eclipte herba and Ligustri lucidi fructus respectively. Our method was more scientific and efficient to control Erzhi formula quality and to avoid false positive results through accurately quantifying wedelolactone and specnuezhenide. Moreover, this method was beneficial for the pharmacokinetics study of EZF.

The production of proteinuria was mainly related to glomerular filtration barrier. Slit diaphragm (SD) was the 

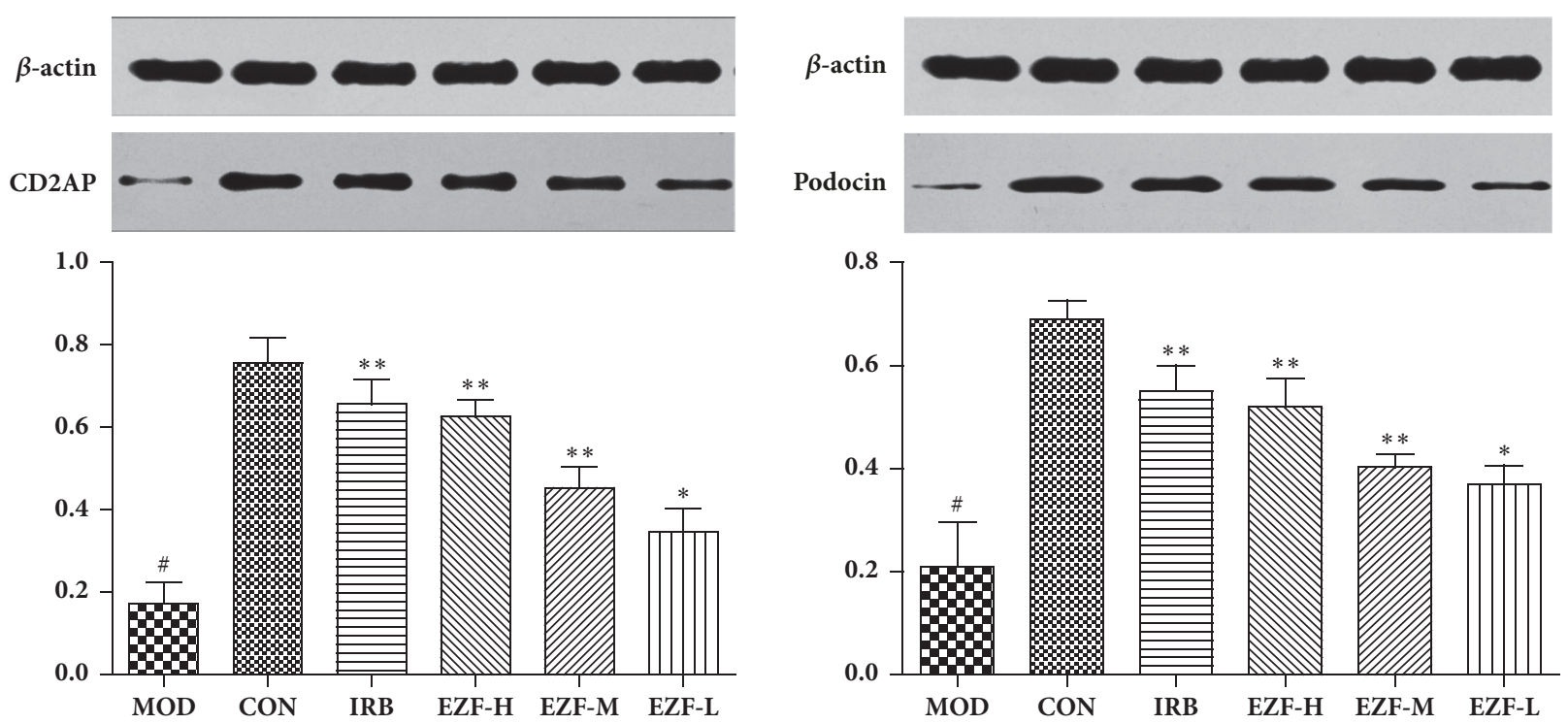

(a)
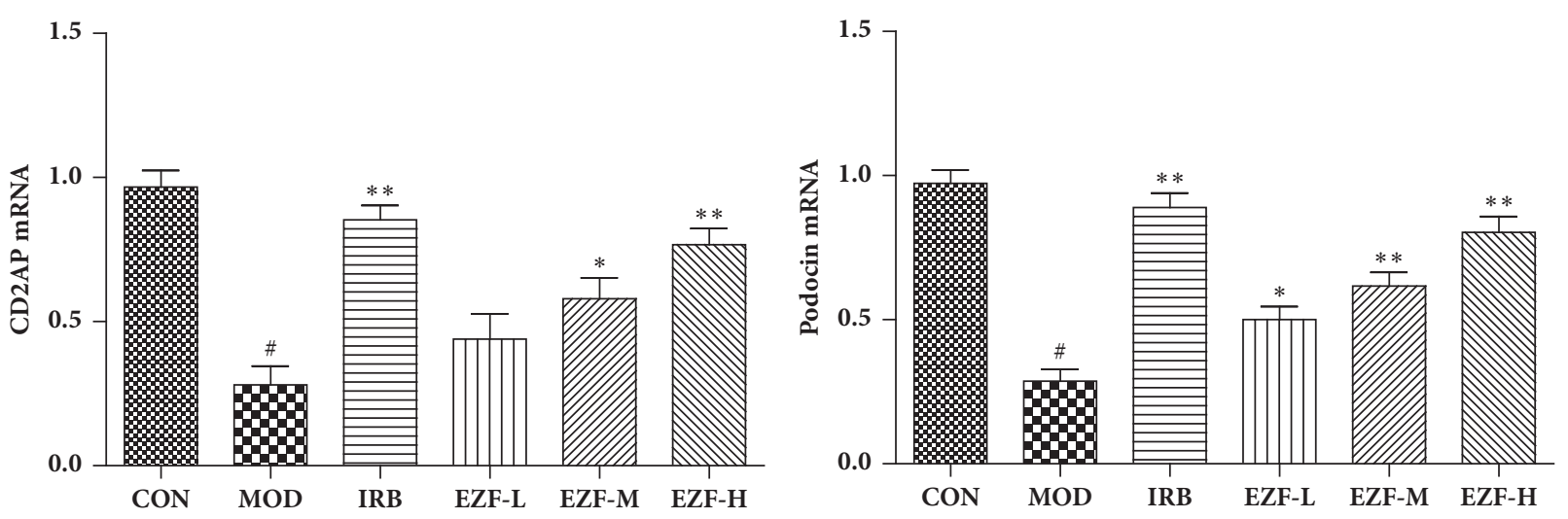

(b)

FIGURE 8: Podocin and CD2AP protein/gene expression in rats renal of each group $(\overline{\boldsymbol{x}}, \mathbf{n}=\mathbf{8})$. (a) Podocin and CD2AP protein expression. (b) Podocin and CD2AP gene expression. Note. \#: compared with Normal group, $\mathrm{P}<0.01$. $* *$ : compared with Model group, $\mathrm{P}<0.01$. $*$ : compared with Model group, $\mathrm{P}<0.05$. Statistical analysis was completed by GraphPad Prism 5.0 software.

final barrier to glomerular filtration and acted as an important role in the development of proteinuria [45]. Studies showed that the nephrin/CD2AP/Podocin complex was necessary to maintain the glomerular filtration function [46]. Podocin was the key functional unit of SD, which had ion channels and signal transduction functions and maintained the structure and function of SD [47]. Podocin also interacted with the intracellular segment of CD2AP and exerted the effect of functional complex by forming lipid raft-like structures [48]. Podocin connected the tight junction protein with the actin of podocyte to exert a scaffolding function which was concentrated on tight junctions of foot processes [49]. Podocin's multiple mutations (V180M, R238S, G92C, etc.) interfered with the structural connectivity of nephron, leading to podocyte pathological changes and massive proteinuria [50]. CD2AP was a transmembrane protein belonging to the immunoglobulin superfamily. Its structure indicated that CD2AP regulated the cytoskeleton and also mediated the interaction of related proteins [51]. When CD2AP expression was decreased, it induced cytoskeletal disruption and podocyte apoptosis [45]. CD2AP had an important role in promoting the binding of $\mathrm{T}$ cell surface antigen $\mathrm{CD} 2$ to antigen-presenting cells [52]. When the function of CD2AP was abnormal, antigen recognition and presentation were not able to be performed which resulted in overimmunization, $\mathrm{T}$ cell death, and proteinuria. Studies reported that the mice of deleting CD2AP gene caused defects in the renal podocyte foot processes, produce large amounts of proteinuria. It also increased the susceptibility of glomerular injury, leading to glomerular sclerosis. Defects in CD2AP caused fusion of the foot processes through deposition of the mesangial matrix, resulting in proteinuria [53-56].

In recent years, studies indicate that $\mathrm{DN}$ was an inflammation-related disease [36]. DN rats produced a variety of inflammatory factors under a high-sugar environment. Inflammatory factors acted on the renal through different 
pathways to accelerate the development of DN [57]. Therefore, the inflammatory response was an important research direction of $\mathrm{DN}$ and was the development of $\mathrm{DN}$ had wide concern, which was an important research direction of DN [58]. TNF- $\alpha$, an inflammatory factor through the NF- $\kappa$ B signaling pathway, activated a variety of inflammatory factors. Moreover, permeability of endothelial cells and production of proteinuria were increased due to TNF-a accumulation and attachment to the glomerular endothelium [59]. IL-6 induced insulin resistance and insulin secretion dysfunction by promoting lipid oxidation and participates in promoting the occurrence and development of DN $[37,60]$.

This experiment successfully replicated DN rats. The results showed that EZF effectively reduced the urinary protein, significantly decreased the IL- $\beta$, TNF- $\alpha$, IL- 6 , and MDA, increased SOD activity, and unregulated the expression of CD2AP, Podocin proteins, and mRNA in renal silt membrane. The renal protective effect of EZF on DN rats was related to upregulate Podocin and CD2AP mRNA/protein expression and reduced renal injury by inhibiting inflammatory reaction. In summary, EZF reversed renal injury in diabetic nephropathy rats by protecting the podocytes and inhibiting the renal inflammation.

High blood glucose level causes the damage of glomerular and podocyte [61]. In this paper, the positive drug, Irbesartan, was not a hypoglycemic drug, but it showed the hypoglycemic effect which deserves further study. In addition, EZF was also not a hypoglycemic drug and its regulation of blood glucose was very weak (Figure 3). However, EZF exhibited a better role in reducing proteinuria, inhibiting renal inflammation and antirenal oxidation, improving glomerular function, and increasing podocyte functional protein. There was no significant difference between Irbesartan and EZF (EZF-H) in improving diabetic nephropathy. These data proved that EZF had a good antidiabetic nephropathy effect. Pharmacokinetic data also showed that the main components of EZF, especially specnuezhenide and Wedelolactone (Table 2), could reach renal tissue and the distribution concentration of specnuezhenide was high. Therefore, we speculated that specnuezhenide was an important substance of EZF in antidiabetic nephropathy. Subsequent studies will explore the molecular mechanism of the antidiabetic nephropathy of specnuezhenide.

\section{Conclusion}

EZF inhibited renal injury and repaired renal function in diabetic rats, which were mediated by protecting the podocytes and upregulating the expression of CD2AP and Podocin.

\section{Data Availability}

The authors agree that others are free to use all the data in the article under reasonable circumstances.

\section{Conflicts of Interest}

All authors had no conflicts of interest regarding the publication of this study.

\section{Authors' Contributions}

Jun Jiang and Jiangning Yin have the same contribution to this paper.

\section{Acknowledgments}

This work was supported by the National Natural Science Foundation of China (no. 81703773), Natural Science Foundation of Jiangsu Province (no. BK20170560), China Postdoctoral Science Foundation (nos. 2016M590424 and 2018T110461), and Jiangsu Postdoctoral Science Foundation (no. 1601184C). The authors gratefully acknowledge the dedicated efforts of the study investigators and staff at the clinical study centers and recognize the participants for their commitment to this trial.

\section{References}

[1] M. S. H. Akash, K. Rehman, and S. Chen, "Effects of coffee on type 2 diabetes mellitus," Nutrition Journal, vol. 30, no. 7-8, pp. 755-763, 2014.

[2] M. S. H. Akash, K. Rehman, and S. Chen, "Spice plant Allium cepa: dietary supplement for treatment of type 2 diabetes mellitus," Nutrition Journal, vol. 30, no. 10, pp. 1128-1137, 2014.

[3] M. S. H. Akash, K. Rehman, M. Tariq, and S. Chen, "Zingiber officinale and type 2 diabetes mellitus: Evidence from experimental studies," Critical Reviews in Eukaryotic Gene Expression, vol. 25, no. 2, pp. 91-112, 2015.

[4] Bu-gao Zhou, Hai-mei Zhao, Xiu-yun Lu et al., "Erzhi Pill ${ }^{\circledR}$ Repairs Experimental Liver Injury via TSC/mTOR Signaling Pathway Inhibiting Excessive Apoptosis," Evidence-Based Complementary and Alternative Medicine, vol. 2017, Article ID 5653643, 15 pages, 2017.

[5] Z.-Y. Li, Q. Li, J. Lü et al., "LC-MS determination and pharmacokinetic study of salidroside in rat plasma after oral administration of suspensions of traditional Chinese medicine Erzhi Wan and Fructus Ligustri lucidi," Journal of Pharmaceutical Analysis, vol. 1, no. 1, pp. 8-12, 2011.

[6] J. C. Milne, P. D. Lambert, S. Schenk et al., "Small molecule activators of SIRT1 as therapeutics for the treatment of type 2 diabetes," Nature, vol. 450, no. 7170, pp. 712-716, 2007.

[7] X.-M. Wan, M. Zhang, P. Zhang et al., "Jiawei Erzhiwan improves menopausal metabolic syndrome by enhancing insulin secretion in pancreatic $\beta$ cells," Chinese Journal of Natural Medicines, vol. 14, no. 11, pp. 823-834, 2016.

[8] E. S. Lee, H. M. Kim, J. S. Kang et al., "Oleanolic acid and Nacetylcysteine ameliorate diabetic nephropathy through reduction of oxidative stress and endoplasmic reticulum stress in a type 2 diabetic rat model," Nephrology Dialysis Transplantation , vol. 31, no. 3, pp. 391-400, 2016.

[9] V. K. Dubey, C. R. Patil, S. M. Kamble et al., "Oleanolic acid prevents progression of streptozotocin induced diabetic nephropathy and protects renal microstructures in Sprague Dawley rats," Journal of Pharmacology and Pharmacotherapeutics, vol. 4, no. 1, pp. 47-52, 2013.

[10] X.-Y. Zeng, Y.-P. Wang, J. Cantley et al., "Oleanolic acid reduces hyperglycemia beyond treatment period with Akt/FoxO1induced suppression of hepatic gluconeogenesis in type-2 diabetic mice," PLoS ONE, vol. 7, no. 7, Article ID e42115, 2012. 
[11] M. A. Baig, V. B. Gawali, R. R. Patil, and S. R. Naik, "Protective effect of herbomineral formulation (Dolabi) on early diabetic nephropathy in streptozotocin-induced diabetic rats," Journal of Natural Medicines, vol. 66, no. 3, pp. 500-509, 2012.

[12] M.-Y. Qi, J.-J. Yang, B. Zhou, D.-Y. Pan, and X. Sun, "Study on the protective effect of ursolic acid on alloxan-induced diabetic renal injury and its underlying mechanisms," Zhongguo ying yong sheng li xue za zhi = Zhongguo yingyong shenglixue zazhi= Chinese journal of applied physiology, vol. 30, no. 5, pp. 445-448, 2014.

[13] C. Ling, L. Jinping, L. Xia, and Y. Renyong, "Ursolic Acid Provides Kidney Protection in Diabetic Rats," Current Therapeutic Research, vol. 75, pp. 59-63, 2013.

[14] Y. Zheng, J. S. Li, X. Zhang, K. Huang, and L. Zheng, "Ursolic acid inhibits early lesions of diabetic nephropathy," International Journal of Molecular Medicine, vol. 26, no. 4, 2010.

[15] J. Wu, X. Ke, W. Fu et al., "Inhibition of Hypoxia-Induced Retinal Angiogenesis by Specnuezhenide, an Effective Constituent of Ligustrum lucidum Ait., through Suppression of the HIF$1 \alpha$ /VEGF Signaling Pathway," Molecules, vol. 21, no. 12, p. 1756, 2016.

[16] A. Hou, M. Q. Tin, and L. Tong, “Toll-like receptor 2-mediated NF-kappa B pathway activation in ocular surface epithelial cells," Eye and Vision, vol. 4, no. 1, 2017.

[17] R. Lindblom, G. Higgins, M. Coughlan, and J. B. De Haan, "Targeting mitochondria-and reactive oxygen species-driven pathogenesis in diabetic nephropathy," Review of Diabetic Studies, vol. 12, no. 1-2, pp. 134-156, 2015.

[18] I. Loeffler and G. Wolf, "Epithelial-to-Mesenchymal Transition in Diabetic Nephropathy: Fact or Fiction?” Cells, vol. 4, no. 4, pp. 631-652, 2015.

[19] Y. S. Kanwar, L. Sun, P. Xie, F. Liu, and S. Chen, "A glimpse of various pathogenetic mechanisms of diabetic nephropathy," Annual Review of Pathology: Mechanisms of Disease, vol. 6, pp. 395-423, 2011.

[20] F. Barutta, F. Piscitelli, S. Pinach et al., "Protective role of cannabinoid receptor type 2 in a mouse model of diabetic nephropathy," Diabetes, vol. 60, no. 9, pp. 2386-2396, 2011.

[21] M. Liu, K. Liang, J. Zhen et al., "Sirt6 deficiency exacerbates podocyte injury and proteinuria through targeting Notch signaling," Nature Communications, vol. 8, no. 1, 2017.

[22] J. Reiser and M. M. Altintas, "Podocytes," F1000Research.

[23] L. Perisic, P. Q. Rodriguez, K. Hultenby et al., "Schip1 Is a Novel Podocyte Foot Process Protein that Mediates Actin Cytoskeleton Rearrangements and Forms a Complex with Nherf2 and Ezrin," PLoS ONE, vol. 10, no. 3, p. e0122067, 2015.

[24] N. Kobayashi, T. Ueno, K. Ohashi et al., "Podocyte injury-driven intracapillary plasminogen activator inhibitor type 1 accelerates podocyte loss via uPAR-mediated $\beta_{1}$ - integrin endocytosis," American Journal of Physiology-Renal Physiology, vol. 308, no. 6, pp. F614-F626, 2015.

[25] H. W. Lee, S. Q. Khan, M. H. Faridi et al., "A podocytebased automated screening assay identifies protective small molecules," Journal of the American Society of Nephrology, vol. 26, no. 11, pp. 2741-2752, 2015.

[26] N. Morito, K. Yoh, M. Ojima et al., "Overexpression of Mafb in podocytes protects against diabetic nephropathy," Journal of the American Society of Nephrology, vol. 25, no. 11, pp. 2546-2557, 2014.

[27] J. Zhu, Y. Chen, M. Yang et al., "Aldosterone is involved in the pathogenesis of obesity-related glomerulopathy through activation of Wnt/ $\beta$-catenin signaling in podocytes," Molecular Medicine Reports, 2018.

[28] J. Wang, Z. Xu, B. Chen, S. Zheng, P. Xia, and Y. Cai, “The role of sirolimus in proteinuria in diabetic nephropathy rats," Iranian Journal of Basic Medical Sciences, vol. 20, no. 12, pp. 1339-1344, 2017.

[29] A. Rubio-Navarro, M. D. Sanchez-Niño, M. Guerrero-Hue et al., "Podocytes are new cellular targets of haemoglobin-mediated renal damage," The Journal of Pathology, vol. 244, no. 3, pp. 296310, 2018.

[30] O. Delézay, Z. He, S. Hodin et al., "Glomerular filtration drug injury: In vitro evaluation of functional and morphological podocyte perturbations," Experimental Cell Research, vol. 361, no. 2, pp. 300-307, 2017.

[31] L. Hao, M. Pan, Y. Zheng, and R. Wang, "Effect of Cordyceps sinensis and Tripterygium wilfordii polyglycosidium on podocytes in rats with diabetic nephropathy," Experimental and Therapeutic Medicine, vol. 7, no. 6, pp. 1465-1470, 2014.

[32] D. Gui, Y. Guo, F. Wang et al., "Astragaloside IV, a novel antioxidant, prevents glucose-induced podocyte apoptosis in vitro and in vivo," PLoS ONE, vol. 7, no. 6, Article ID e39824, 2012.

[33] E. Arif, Y. S. Rathore, B. Kumari et al., "Slit diaphragm protein nephl and its signaling: A novel therapeutic target for protection of podocytes against: Glomerular injury," The Journal of Biological Chemistry, vol. 289, no. 14, pp. 9502-9518, 2014.

[34] E. Arif, M. C. Wagner, D. B. Johnstone et al., "Motor protein Myolc is a podocyte protein that facilitates the transport of slit diaphragm protein Nephl to the podocyte membrane," Molecular and Cellular Biology, vol. 31, no. 10, pp. 2134-2150, 2011.

[35] Y. Matsushita, D. Ogawa, J. Wada et al., "Activation of peroxisome proliferator-activated receptor $\delta$ inhibits streptozotocininduced diabetic nephropathy through anti-inflammatory mechanisms in mice," Diabetes, vol. 60, no. 3, pp. 960-968, 2011.

[36] R. Mohamed, C. Jayakumar, F. Chen et al., "Low-dose IL-17 therapy prevents and reverses diabetic nephropathy, metabolic syndrome, and associated organ fibrosis," Journal of the American Society of Nephrology, vol. 27, no. 3, pp. 745-765, 2016.

[37] A. K. H. Lim and G. H. Tesch, "Inflammation in Diabetic Nephropathy," Mediators of Inflammation, vol. 2012, Article ID 146154, 12 pages, 2012.

[38] D. T. Skelly, E. Hennessy, M.-A. Dansereau, and C. Cunningham, "A systematic analysis of the peripheral and CNS effects of systemic LPS, IL- $1 \beta$, [corrected] TNF- $\alpha$ and IL- 6 challenges in C57BL/6 mice," PLoS ONE, vol. 8, no. 7, Article ID e69123, 2013.

[39] X. Su, Y. He, W. Yang, Y. Wang, W. Zhang, and Y. Wang, "Effect of dan hong injection on PON1, SOD activity and mda levels in elderly patients with coronary heart disease," International Journal of Clinical and Experimental Medicine, vol. 7, no. 12, pp. 5886-5889, 2014.

[40] Z. J. Xu, S. Shu, Z. J. Li, Y. M. Liu, R. Y. Zhang, and Y. Zhang, "Liuwei Dihuang pill treats diabetic nephropathy in rats by inhibiting of TGF- $\beta$ /SMADS, MAPK, and NF-kB and upregulating expression of cytoglobin in renal tissues," Medicine, vol. 96, no. 3, p. e5879, 2017.

[41] X. Chen, R. Wu, Y. Kong et al., “Tanshinone II Aattenuates renal damage in STZ-induced diabetic rats via inhibiting oxidative stress and inflammation," Oncotarget , vol. 8, no. 19, pp. 3191531922, 2017.

[42] M. V. Malishevskii and M. V. Malishevskiŭ, "Irbesartan in clinical practice," Kardiologiia, vol. 52, no. 11, pp. 66-74, 2012. 
[43] C. Wang, C. Min, X. Rong, T. Fu, X. Huang, and C. Wang, "Irbesartan can improve blood lipid and the kidney function of diabetic nephropathy," Discovery Medicine, vol. 20, no. 108, pp. 67-77, 2015.

[44] S. Roselli, L. Heidet, M. Sich et al., "Early glomerular filtration defect and severe renal disease in podocin-deficient mice," Molecular and Cellular Biology, vol. 24, no. 2, pp. 550-560, 2004.

[45] P. Saurus, T. A. Tolvanen, S. Lindfors et al., "Inhibition of SHIP2 in CD2AP-deficient podocytes ameliorates reactive oxygen species generation but aggravates apoptosis," Scientific Reports, vol. 7, no. 1, 2017.

[46] M. Yi, L. Zhang, Y. Liu et al., "Autophagy is activated to protect against podocyte injury in adriamycin-induced nephropathy," American Journal of Physiology-Renal Physiology, vol. 313, no. 1, pp. F74-F84, 2017.

[47] M. M. Rinschen, P. Bharill, X. Wu et al., "The ubiquitin ligase Ubr4 controls stability of podocin/MEC-2 supercomplexes," Human Molecular Genetics, vol. 25, no. 7, pp. 1328-1344, 2016.

[48] L. Barisoni and J. B. Kopp, "Update in podocyte biology: Putting one's best foot forward," Current Opinion in Nephrology and Hypertension, vol. 12, no. 3, pp. 251-258, 2003.

[49] S. Baijnath, S. Murugesan, I. Mackraj, P. Gathiram, and J. Moodley, "The effects of sildenafil citrate on urinary podocin and nephrin mRNA expression in an l-NAME model of preeclampsia," Molecular and Cellular Biochemistry, vol. 427, no. 12, pp. 59-67, 2017.

[50] B. Sutariya and M. Saraf, " $\alpha$-asarone reduce proteinuria by restoring antioxidant enzymes activities and regulating necrosis factor $\kappa \mathrm{B}$ signaling pathway in doxorubicin-induced nephrotic syndrome," Biomedicine \& Pharmacotherapy, vol. 98, pp. 318$324,2018$.

[51] K. Tsuji, T. G. Păunescu, H. Suleiman et al., "Recharacterization of the Glomerulopathy in CD2AP Deficient Mice by High-Resolution Helium Ion Scanning Microscopy," Scientific Reports, vol. 7, no. 1, 2017.

[52] K. Badour, J. Zhang, F. Shi et al., "The Wiskott-Aldrich syndrome protein acts downstream of CD2 and the CD2AP and PSTPIP1 adaptors to promote formation of the immunological synapse," Immunity, vol. 18, no. 1, pp. 141-154, 2003.

[53] S.-H. Kwon, S. Oh, M. Nacke, K. E. Mostov, and J. H. Lipschutz, "Adaptor protein CD2AP and L-type lectin LMAN2 regulate exosome cargo protein trafficking through the Golgi complex," The Journal of Biological Chemistry, vol. 291, no. 49, pp. 2546225475, 2016.

[54] H.-Y. Park, S.-B. Seong, S.-Y. Min, and T.-S. Ha, "CD2associated protein/phosphoinositide 3-kinase signaling has a preventive role in angiotensin II-induced podocyte apoptosis," The International Journal of Biochemistry \& Cell Biology, vol. 79, pp. 370-381, 2016.

[55] T. D. Cummins, K. Z. L. Wu, and P. Bozatz, "PAWS1 controls cytoskeletal dynamics and cell migration through association with the SH3 adaptor CD2AP," Journal of Cell Science, vol. 131, no. 1, Article ID 202390, 2018.

[56] Haiyan $\mathrm{Xu}, \mathrm{Xu}$ Wang, Mingming Liu, and Xueyuan He, "Tangzhiqing Granules Alleviate Podocyte EpithelialMesenchymal Transition in Kidney of Diabetic Rats," Evidence-Based Complementary and Alternative Medicine, vol. 2017, Article ID 1479136, 9 pages, 2017.

[57] Y. Sawa, S. Takata, Y. Hatakeyama, H. Ishikawa, and E. Tsuruga, "Expression of toll-like receptor 2 in glomerular endothelial cells and promotion of diabetic nephropathy by
Porphyromonas gingivalis lipopolysaccharide," PLOS ONE, vol. 9, no. 5, 2014.

[58] J. Donate-Correa, E. Martín-Núñez, M. Muros-de-Fuentes, C. Mora-Fernández, and J. F. Navarro-González, "Inflammatory Cytokines in Diabetic Nephropathy," Journal of Diabetes Research, vol. 2015, Article ID 948417, 9 pages, 2015.

[59] J. F. Navarro, C. Mora, M. Muros, and J. García, "Urinary tumour necrosis factor-alpha excretion independently correlates with clinical markers of glomerular and tubulointerstitial injury in type 2 diabetic patients," Nephrology Dialysis Transplantation, vol. 21, no. 12, pp. 3428-3434, 2006.

[60] K. Rehman and M. S. H. Akash, "Mechanisms of inflammatory responses and development of insulin resistance: How are they interlinked?" Journal of Biomedical Science, vol. 23, no. 1, article no. 87, 2016.

[61] J. Lei, L. Zhao, Y. Zhang, Y. Wu, and Y. Liu, "High GlucoseInduced Podocyte Injury Involves Activation of Mammalian Target of Rapamycin (mTOR)-Induced Endoplasmic Reticulum (ER) Stress," Cellular Physiology and Biochemistry, vol. 45, no. 6, pp. 2431-2443, 2018. 


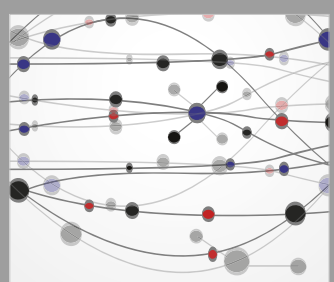

The Scientific World Journal
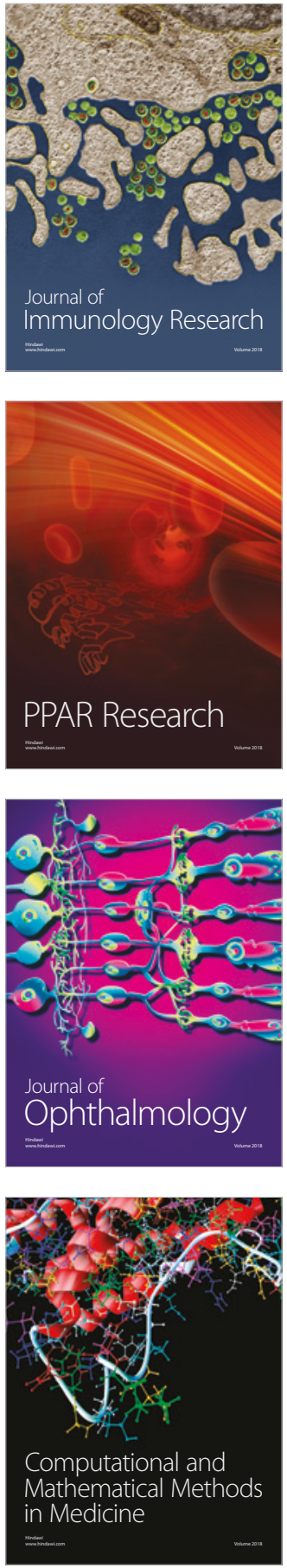

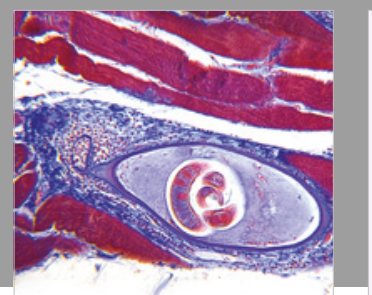

Gastroenterology Research and Practice

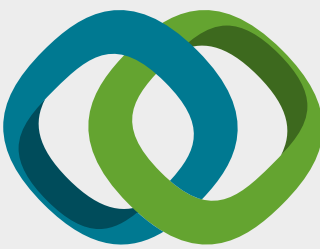

\section{Hindawi}

Submit your manuscripts at

www.hindawi.com
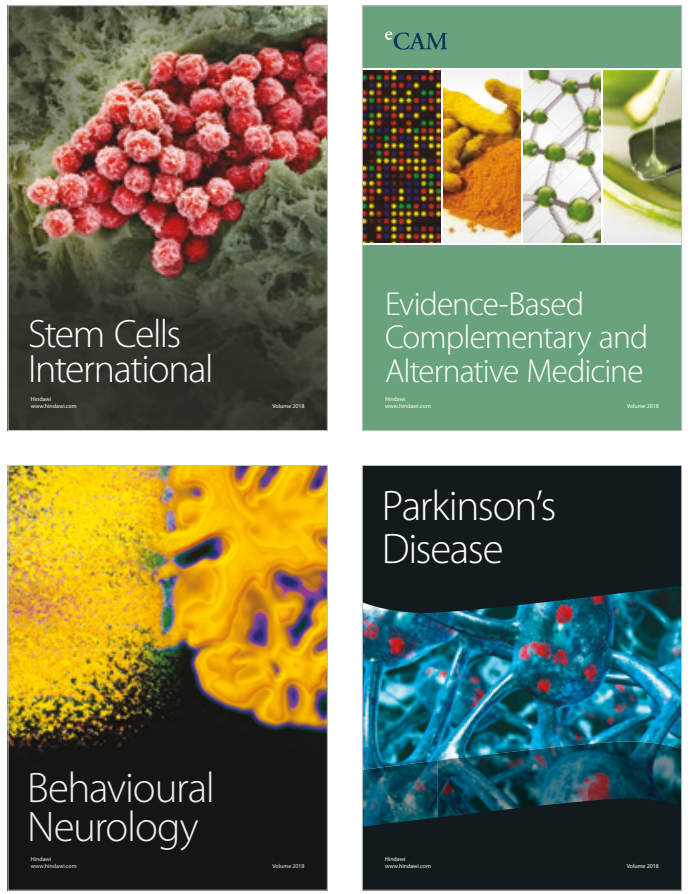

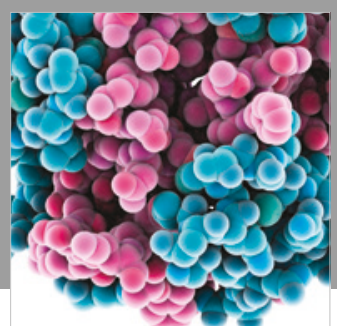

ournal of

Diabetes Research

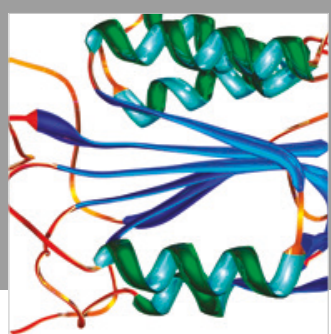

Disease Markers
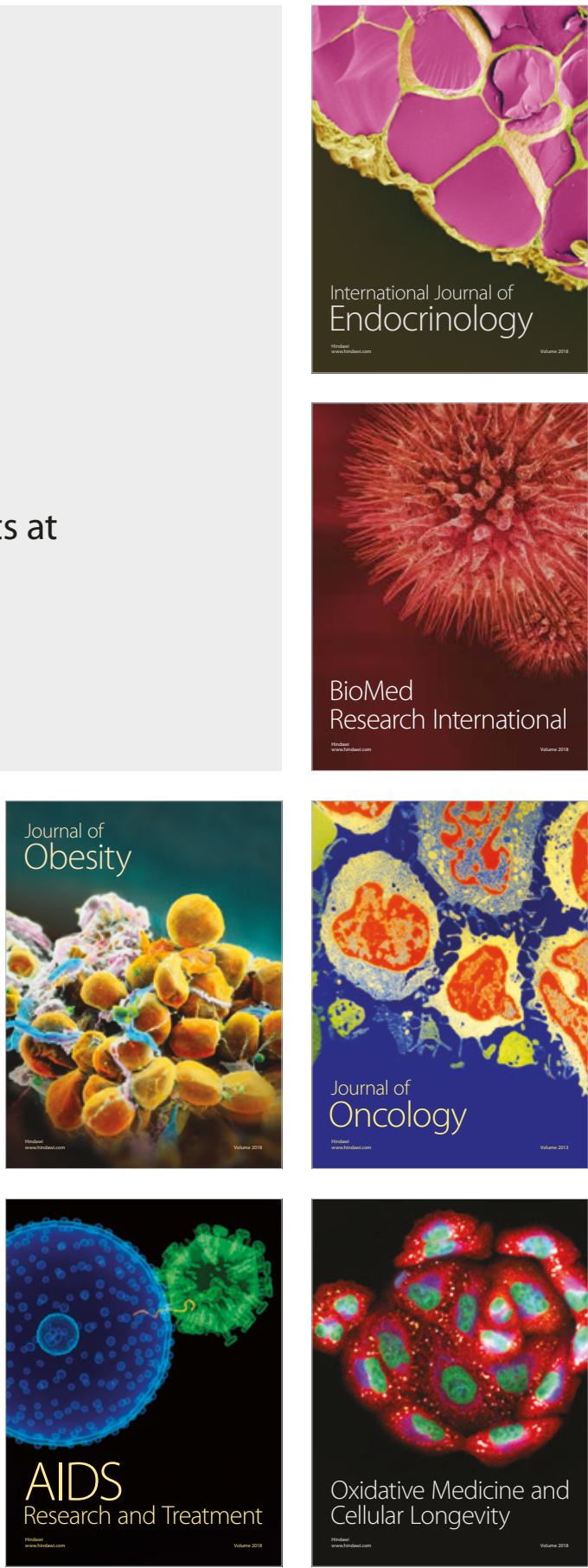